\title{
Preparation of Partially Deuterium-labeled Poly(4-trimethylsilylstyrene)s and Unperturbed Dimensions in Bulk
}

\author{
Masashi Harada, ${ }^{\dagger \dagger} \dagger^{\dagger \dagger}$ Takahisa Suzuki, ${ }^{\dagger \dagger}$ Masayuki OHYa, Atsushi TAKano, and Yushu Matsushita \\ Department of Applied Chemistry, Nagoya University, Furo-cho, Chikusa-ku, Nagoya 464-8603, Japan
}

(Received February 5, 2004; Accepted April 9, 2004; Published July 15, 2004)

\begin{abstract}
Monodisperse poly(4-timethylsilylstyrene)s (PTMSS's) and the partially deuterium-labeled polymers were prepared and characterized to determine their unperturbed dimensions by measuring small-angle neutron scattering of their blends. For polymer pairs with three different molecular weight $\left(M_{\mathrm{w}}\right)$, the radii of gyration $\left(R_{\mathrm{g}}\right)$ in bulk were estimated and a power law was deduced as $R_{\mathrm{g}}=0.022 M_{\mathrm{w}}{ }^{0.50}(\mathrm{~nm})$. The power index 0.50 was consistent with the theoretical prediction for ideal chains, $1 / 2$. Although the prefactor, 0.022 , is apparently smaller than that of polystyrene (PS), 0.0275 , the segment length of this polymer, $0.72 \mathrm{~nm}$, is similar to that of PS, $0.69 \mathrm{~nm}$. It is indicated that the bulky trimethylsilyl group on 4-position does not affect much on the chain conformation of polystyrene backbone. [DOI 10.1295/polymj.36.538]

KEY WORDS Poly(4-trimethylsilylstyrene) / Unperturbed Dimension / Small-angle Neutron Scattering /
\end{abstract}

Poly(4-trimethylsilylstyrene) (PTMSS) has been studied for applications to separation membranes and bilayer resists because it has high permeability to gases ${ }^{1,2}$ and high durability against $\mathrm{O}_{2}$-plasma etching. ${ }^{3,4}$

Conventionally PTMSS was synthesized free-radically. It was reported that 4-trimethylsilylstyrene can be polymerized anionically ${ }^{5}$ and living-radically. ${ }^{6}$ With these polymerization techniques we can design polymer architecture and prepare block copolymers and cyclic polymers containing PTMSS, useful to develop higher-performance materials.

Recently experimental evidence showing a single phase from polymer blends of PTMSS and polyisoprene (PI) has been found by TEM observation, glass transition temperature measurements and optical microscopy. This suggests that PTMSS and PI are miscible, which is unpredictable because their chemical structures are so different that no attractive force between the groups was expected.

Miscibility behavior of polymer blends is in general expressed with $\chi$ parameter. Since it can be precisely determined by measurement of small-angle neutron scattering (SANS $)^{7,8}$ in conjunction with the analysis based on the random phase approximation (RPA), ${ }^{9}$ the radius of gyration $\left(R_{\mathrm{g}}\right)$ of PTMSS should be known in advance of the study on miscibility of blends. The radii of gyration of polymers in bulk can be estimated by measuring SANS coherent scattering intensity from blends of normal hydrogenated polymers with the deu- terium-labeled counterpart. Therefore, PTMSS has to be deuterated. Partially deuterium-labeled polymers ( $d$-PTMSS) together with normal hydrogenated ones (PTMSS) were synthesized and characterized in this work. Successively unperturbed dimensions of these polymers were measured by SANS.

\section{EXPERIMENTAL}

\section{Monomer Synthesis}

Partially deuterium-labeled 4-trimethylsilylstyrene was synthesized by reaction of deuterated chlorotrimethylsilylsilane purchased from CDN Isotopes with Grignard reagent derived from 4-chlorostyrene of Hokko Chemical Industry Co., Ltd. ${ }^{10}$ Tetrahydrofuran (THF) with non-stabilizer of Hayashi Pure Chemical Ind., Co., Ltd. was used as dry solvent and experiments were performed under nitrogen gas atmosphere. 4-Chlorostyrene ( $150 \mathrm{~g}, 1.1 \mathrm{~mol})$ was first dissolved in THF $(130 \mathrm{~mL})$ and the solution was dropped very slowly onto activated $\mathrm{Mg}$ turnings $(31.8 \mathrm{~g}, 1.3 \mathrm{~mol})$ of Nacalai Tesque Inc. The solution was then refluxed for $20 \mathrm{~min}$ at about $65^{\circ} \mathrm{C}$ and cooled to room temperature. Successively neat deuterated chlorotrimethylsilane $(100 \mathrm{~mL}, 0.8 \mathrm{~mol})$ was added dropwisely onto THF solution of the Grignard reagent and the solution was stirred for $1 \mathrm{~h}$ at room temperature, followed by neutralization and extraction. After solvents were evaporated from organic phase, crude partially deuterium-labeled 4-trimethylsilylstyrene was obtained by

\footnotetext{
${ }^{\dagger}$ To whom correspondence should be addressed (E-mail: harada@mosk.tytlabs.co.jp).

${ }^{\dagger}$ Present Address: Toyota Central R\&D Labs., Inc., Nagakute-cho, Aichi 480-1192, Japan

${ }^{\dagger \dagger}$ Present Address: JSR Co., Yokkaichi, Mie 510-8552, Japan
} 
distillation under reduced pressure twice. The yield was $97 \mathrm{~g}$, the conversion being $77 \%$. ${ }^{1} \mathrm{H}$ NMR spectra were obtained with UNITY INOVA 500 of Varian Inc. at $500 \mathrm{MHz}$ at room temperature in $\mathrm{CDCl}_{3}$. Chemical shifts were referred to residual $\mathrm{CHCl}_{3}$ in $\mathrm{CDCl}_{3} .{ }^{1} \mathrm{H}$ NMR: $\delta \quad 7.38-7.49(\mathrm{~m}, 4 \mathrm{H}), 6.72(\mathrm{q}$, $1 \mathrm{H}), 5.23-5.79(\mathrm{~m}, 2 \mathrm{H})$. It was confirmed by gas chromatography that $0.4 \%$ of 4-chlorostyrene was still present in carefully distilled monomer.

Normal hydrogenated 4-trimethylsilylstyrene monomer was also synthesized in almost the same manner as partially deuterium-labeled monomer using chlorotrimethylsilane instead of deuterated chlorotrimethylsilane. ${ }^{1} \mathrm{H}$ NMR: $\delta 0.34$ (s, 9H, $\left.\mathrm{Si}\left(\mathrm{CH}_{3}\right)_{3}\right), 7.45-7.57$ $(\mathrm{m}, 4 \mathrm{H}), 6.78(\mathrm{q}, 1 \mathrm{H}), 5.30-5.86(\mathrm{~m}, 2 \mathrm{H})$.

\section{Preparation and Characterization of Polymers}

Anionic polymerization reactions were carried out under high vacuum in sealed glass reactors with break seals. Partially deuterium-labeled 4-trimethylsilylstyrene was dried over calcium hydride and purified with triphenylmethyllithium. The evacuated and isolated glass apparatus for polymerization was rinsed with $n$-butyllithium in benzene. Sec-butyllithium as initiator and purified THF as solvent in different ampules were introduced into reaction vessels and polymerization reactions for both deuterium-labeled and hydrogenated monomers were carried out for $0.5-5 \mathrm{~h}$ at $-78^{\circ} \mathrm{C}$ in almost the same manner as reported previously. ${ }^{11}$ After the living polymers were quenched with isopropanol, the polymers were precipitated in excess methanol and freeze-dried with benzene. Fractionation by GPC had to be carried out for higher molecular weight $d$-PTMSS because of a certain degree of polymer contamination with low molecular weight due to small amount of residual 4-chlorostyrene in the synthesized monomer. Infrared (IR) spectra were obtained at room temperature with Herschel FT/IR230 of Jasco Inc. For $d$-PTMSS, ${ }^{1} \mathrm{H}$ NMR: $\delta 7.38$ $7.48(\mathrm{~m}, 4 \mathrm{H}), 6.71(\mathrm{q}, 1 \mathrm{H}), 5.25-5.77(\mathrm{~m}, 2 \mathrm{H}) . \mathrm{IR}$ $\left(\mathrm{cm}^{-1}\right)$ : 714 ( $\mathrm{Si}-\mathrm{C}$ stretch), $995\left(\mathrm{Si}-\mathrm{CH}_{3}\right.$ deformation), 2210 (Si-C stretch). For PTMSS, ${ }^{1} \mathrm{H}$ NMR: $\delta$ $0.34\left(\mathrm{~s}, 9 \mathrm{H}, \mathrm{Si}\left(\mathrm{CH}_{3}\right)_{3}\right), 7.45-7.55(\mathrm{~m}, 4 \mathrm{H}), 6.78(\mathrm{q}$, $1 \mathrm{H}), \quad 5.32-5.84(\mathrm{~m}, 2 \mathrm{H})$. IR $\left(\mathrm{cm}^{-1}\right): 852(\mathrm{Si}-\mathrm{C}$ stretch), 1248 ( $\mathrm{Si}-\mathrm{CH}_{3}$ deformation), 2903 (Si-C stretch).

Molecular weights were determined by multiangle laser light scattering (MALLS) using DAWN EOS of Wyatt Technology Co. with $d n / d c$ of 0.138 and 0.143 for $d$-PTMSS and PTMSS, respectively. Molecular weight distribution was estimated with gel permeation chromatography (GPC) using a set of three $\mathrm{G} 4000 \mathrm{H}_{\mathrm{HR}}$ columns of Tosoh Co. at $38^{\circ} \mathrm{C}$ with THF as eluting solvent at a flow rate of $1 \mathrm{~mL} / \mathrm{min}$. Densities of polymers were measured with gas dis- placement technique using helium gas at $10 \mathrm{psi}$ on Ultra Pycnometer 1000 of Quantachrome Co. Differential scanning calorimetry (DSC) data were obtained at a heating rate of $10^{\circ} \mathrm{C} / \mathrm{min}$ under nitrogen atmosphere with Exstar 6000 of Seiko Instruments Inc. Thermal gravimetric analysis (TGA) was performed at a heating rate of $10^{\circ} \mathrm{C} / \mathrm{min}$ under argon atmosphere on Thermo Plus 2 of Rigaku Co.

\section{SANS Measurements}

Blend films were prepared by casting from toluene solutions of PTMSS and $d$-PTMSS (50/50 by w/w) for several days and the films were placed in quartz cells with $2 \mathrm{~mm}$ thickness and sealed under vacuum, followed by annealing at $170^{\circ} \mathrm{C}$. Small-angle neutron scattering was measured using SANS-U spectrometer of Neutron Science Laboratory at the Institute for Solid State Physics, the University of Tokyo installed at JRR-3M of the Japan Atomic Energy Research Institute in Tokai, Japan. All measurements were performed at $170^{\circ} \mathrm{C}$ for $3 \mathrm{~h}$. The wavelength of the neutron beam was $0.7 \mathrm{~nm}$, the sample-to-detector distance was held at $8 \mathrm{~m}$ and the pinhole with $5 \mathrm{~mm}$ in diameter was set just upstream of the sample. SANS intensity was determined with a 2D detector and averaged circularly. Incoherent scattering were calibrated with the intensity of normal hydrogenated poly(4-trimethylsilylstyrene) measured as above assuming additivity of volume. ${ }^{12}$

\section{RESULTS AND DISCUSSION}

Partially deuterium-labeled 4-trimethylsilylstyrene and hydrogenated polymers were polymerized anionically to give polymers with different molecular weight. The weight average molecular weight, $M_{\mathrm{w}}$, and polydispersity index, $M_{\mathrm{w}} / M_{\mathrm{n}}$, are shown in Table I. Three pairs of polymers with similar molecular weight were obtained to prepare three polymer blends for SANS measurement and polydispersity indices of all polymers were small enough. The molecular weight of the blend was defined as the average of the degree of polymerization. ${ }^{13}$

Table I. Molecular characteristics of poly(4-trimethylsilylstyrene)s and partially deuterium-labeled polymers

\begin{tabular}{ccccc}
\hline Polymer Code & $10^{-3} M_{\mathrm{w}}{ }^{\mathrm{a}}$ & $M_{\mathrm{w}} / M_{\mathrm{n}}{ }^{\mathrm{b}}$ & Blend Code & $10^{-3} M_{\mathrm{w}}{ }^{\mathrm{c}}$ \\
\hline H-35 & 35.0 & 1.02 & HD-34 & 33.7 \\
D-34 & 33.8 & 1.02 & & \\
\hline H-47 & 47.4 & 1.02 & HD-44 & \multirow{2}{*}{44.0} \\
D-43 & 42.6 & 1.07 & & \\
\hline H-68 & 68.3 & 1.01 & HD-68 & \multirow{2}{*}{67.6} \\
D-70 & 70.2 & 1.03 & & \\
\hline
\end{tabular}

${ }^{a}$ Determined by MALLS. ${ }^{b}$ Determined by GPC. ${ }^{c}$ Calculated average value using the method in Ref 13. 
Table II. Properties of poly(4-trimethylsilylstyrene)s (PTMSS) and partially deuterium-labeled polymers ( $d$-PTMSS)

\begin{tabular}{lccc}
\hline Polymer & $\begin{array}{c}\rho^{\mathrm{a}} \\
\left(\mathrm{g} / \mathrm{cm}^{3}\right)\end{array}$ & $\begin{array}{c}T_{\mathrm{g}}^{\mathrm{b}} \\
\left({ }^{\circ} \mathrm{C}\right)\end{array}$ & $\begin{array}{c}T_{\mathrm{d}}{ }^{\mathrm{c}} \\
\left({ }^{\circ} \mathrm{C}\right)\end{array}$ \\
\hline PTMSS & 0.963 & 134 & 341 \\
$d$-PTMSS & 1.006 & 133 & 376 \\
\hline
\end{tabular}

${ }^{a}$ Measured with pycnometric gas displacement technique. ${ }^{b}$ Measured with DSC. ${ }^{c}$ Measured with TGA.

The density of PTMSS and that of $d$-PTMSS are shown in Table II. The density ratio of the polymer, $0.963 / 1.006=0.95_{7}$, is consistent with the ratio of monomer molecular weight, $176 / 185=0.95_{1}$, indicating that the extent of monomer packing was unchanged by deuterium-labeling. Glass transition temperature $\left(T_{\mathrm{g}}\right)$ and thermal degradation temperature $\left(T_{\mathrm{d}}\right)$ are listed in Table II. Because the temperature for SANS measurement, $170^{\circ} \mathrm{C}$, is above $T_{\mathrm{g}}$ and much below $T_{\mathrm{d}}$, the blend sample is assured to be in the melt and stable state during SANS measurement.

Figure 1 shows the SANS coherent intensity for the three polymer blends measured at $170^{\circ} \mathrm{C}$ as the functions of the scattering vector $q(=4 \pi \sin \theta / \lambda)$, where $2 \theta$ is the scattering angle and $\lambda$ denotes wavelength of neutron. The intensity $(I)$ at low scattering vector (q) regime increased with $M_{\mathrm{w}}$, indicating the expansion of the polymer coils in accordance with chain length. To estimate $R_{\mathrm{g}}$ of the polymers, Guinier plots of $\ln I$ vs. $q^{2}$ were made as shown in Figure 2. In the Guinier region defined as $q^{2}<1.3^{2} / R_{\mathrm{g}}{ }^{2}$ the plots were aligned on straight lines and $R_{\mathrm{g}}$ was determined for each profile by equating the slop as $-1 / 3 R_{\mathrm{g}}{ }^{2} \cdot{ }^{14} \mathrm{In}$ Figure 3 the $R_{\mathrm{g}}$ is plotted double-logarithmically against $M_{\mathrm{w}}$ assuming a power law behavior. The line fitting gives the relationship, $R_{\mathrm{g}}=0.022 M_{\mathrm{w}}{ }^{0.50}(\mathrm{~nm})$. The index 0.50 is consistent with the theoretically-predicted value of $1 / 2,{ }^{15}$ confirmed experimentally for several polymers in bulk. ${ }^{16,17}$ From the prefactor, 0.022 , the segment length $(b)$, defined as $R_{\mathrm{g}}=$

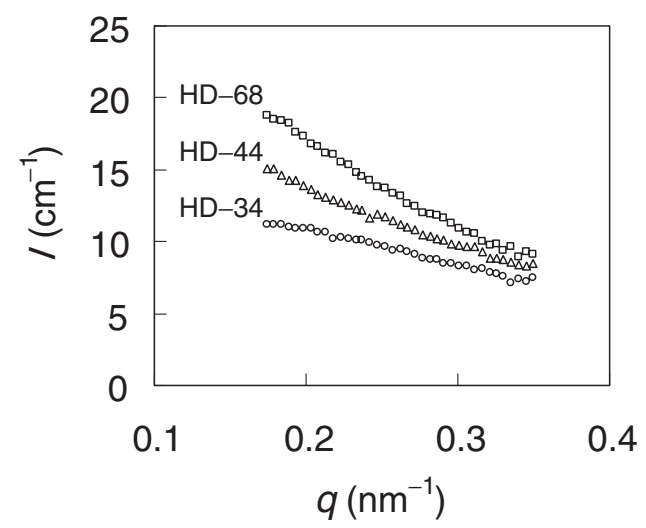

Figure 1. Coherent scattering intensity vs. scattering vector

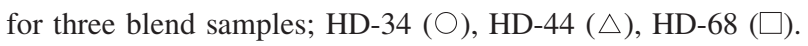

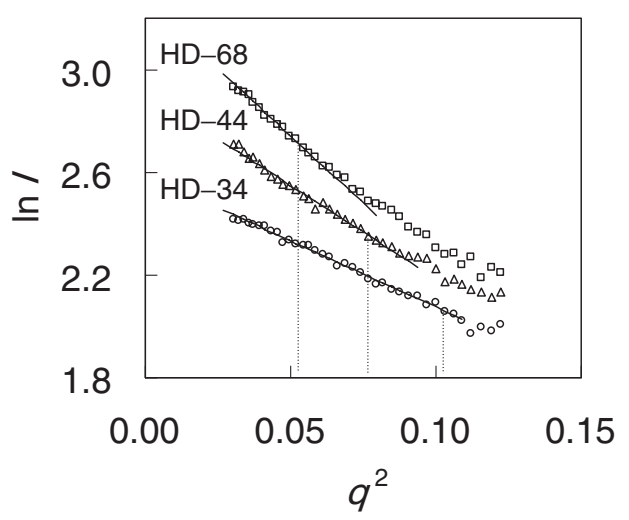

Figure 2. Guinier plots of the data in Figure 1; HD-34 $(\bigcirc)$, HD-44 $(\triangle)$, HD-68 $(\square)$. Linier fittings are shown by solid lines and the Guinier ranges are indicated by dotted lines.

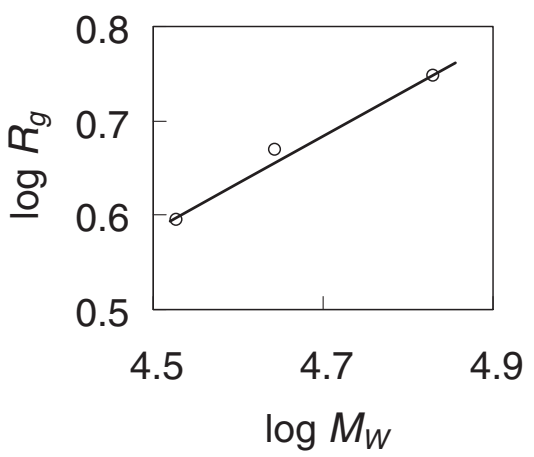

Figure 3. Double-logarithmic plots of $R_{\mathrm{g}} v s . M_{\mathrm{w}}$ for three blend samples. The linear fitting is shown by a solid line: $\log R_{\mathrm{g}}=0.50 \log M_{\mathrm{w}}-1.66$.

$b(N / 6)^{1 / 2}$ where $N$ is the degree of polymerization, was estimated as $0.72 \mathrm{~nm}$. This is similar to that of PS, $0.69 \mathrm{~nm}$ and thus the monomer units of a PTMSS chain distributed in almost the same way as that of a PS chain irrespective of the existence of fairly large trimethylsilyl group on 4-position.

$\chi$ parameter between PTMSS and PI can be estimated precisely using $R_{\mathrm{g}}$ obtained in this study. SANS measurement for $d$-PTMSS/PI blends is now in progress.

\section{CONCLUSIONS}

Partially deuterium-labeled 4-trimethylsilylstyrene ( $d$-TMSS) together with hydrogenated TMSS was synthesized. The monomers were polymerized anionically and it was confirmed that three pairs of $d$ PTMSS and PTMSS were monodisperse by careful molecular characterization. SANS measurement was performed for three pairs of samples to obtain $R_{\mathrm{g}}$ 's in bulk state. The relationship in between $R_{\mathrm{g}}$ and $M_{\mathrm{w}}$ was obtained as $R_{\mathrm{g}}=0.022 M_{\mathrm{w}}{ }^{0.50}(\mathrm{~nm})$, which is essentially equivalent to that for polystyrene in 
bulk, $R_{\mathrm{g}}=0.0275 M_{\mathrm{w}}{ }^{1 / 2}(\mathrm{~nm})$, indicating that the trimethylsilyl group on 4-position does not significantly affect polymer chain conformation of the PS backbone.

Acknowledgment. This work was partially supported by the Ministry of Education, Culture, Sports, Science and Technology, Japan, Grant-in-Aid program \#13031040. This work was also done under the support of COE program entitled "The Creation of Nature-Guided Materials Processing" in the school of Engineering, Nagoya University, Nagoya, Japan, which has been assigned as one of the 21st century COE programs. This research was supported in part by a grant from Daiko Foundation.

\section{REFERENCES}

1. Y. Kawakami, H. Karasawa, T. Aoki, Y. Yamamura, H. Hisada, and Y. Yamashita, Polym. J., 17, 1159 (1985).

2. V. S. Khotimskii, V. G. Filippova, I. S. Bryantseva, V. I. Bondar, V. P. Shantaovich, and Y. P. Yampolskii, J. Appl. Polym. Sci., 78, 1612 (2000).

3. M. Suzuki, K. Saigo, H. Gokan, and Y. Ohnishi, J. Electrochem. Soc., 130, 1962 (1983).

4. S. A. MacDonald, H. Ito, and C. G. Willson, Microelectron.
Eng., 1, 269 (1983).

5. A. Hirao and S. Nakahama, Prog. Polym. Sci., 17, 283 (1992).

6. B. W. McQuillan and S. Paguio, Fusion Technol., 38, 108 (2000).

7. M. Shibayama, H. Yang, R. S. Stein, and C. C. Han, Macromolecules, 18, 2179 (1985).

8. D. Y. Ryu, M. S. Park, S. H. Chae, J. Jang, J. K. Kim, and T. P. Russell, Macromolecules, 35, 8676 (2002).

9. P. G. de Gennes, "Scaling Concepts in Polymer Physics," Cornell University Press, Ithaca, N.Y., 1978.

10. P. Chaumont, G. Beinert, J. E. Herz, and P. Rempp, Makromol. Chem., 183, 1181 (1982).

11. Y. Matsushita, K. Shimizu, T. Nakao, H. Choshi, I. Noda, and M. Nagasawa, Polym. J., 18, 361 (1986).

12. M. Seki, H. Nakano, S. Yamauchi, J. Suzuki, and Y. Matsushita, Macromolecules, 32, 3227 (1999).

13. F. Boué, M. Nierlich, and L. Leibler, Polymer, 23, 29 (1982).

14. A. Guinier and G. Fournet, "Small-Angle Scattering of X-Rays,” Wiley, New York, N.Y., 1955.

15. P. J. Flory, "Principles of Polymer Chemistry," Cornell University Press, Ithaca, N.Y., 1953.

16. J. P. Cotton, H. Benoit, B. Farnoux, J. Higgins, G. Jannink, R. Ober, C. Picot, and J. des Cloizeaux, Macromolecules, 7, 863 (1974).

17. R. G. Kirste, W. A. Kruse, and K. Ibel, Polymer, 16, 120 (1975). 